\title{
First-principles characterization of single-electron polaron in $\mathrm{WO}_{3}$
}

\author{
Eric Bousquet, ${ }^{1}$ Hanen Hamdi $\odot,{ }^{1}$ Pablo Aguado-Puente $\odot,{ }^{2}$ Ekhard K. H. Salje, ${ }^{3}$ \\ Emilio Artacho $\oplus^{4,5,6}$ and Philippe Ghosez $\oplus^{1}$ \\ ${ }^{1}$ CESAM-QMAT Physique Théorique des Matériaux, Université de Liège, B-4000 Sart-Tilman, Belgium \\ ${ }^{2}$ School of Mathematics and Physics, Queen's University Belfast, Belfast BT7 1NN, Northern Ireland, United Kingdom \\ ${ }^{3}$ Department of Earth Sciences, University of Cambridge, Cambridge CB2 3EQ, United Kingdom \\ ${ }^{4}$ Theory of Condensed Matter, Cavendish Laboratory, University of Cambridge, Cambridge CB3 OHE, United Kingdom \\ ${ }^{5}$ CIC Nanogune and DIPC, Tolosa Hiribidea 76, E-20018 San Sebastián, Spain \\ ${ }^{6}$ Ikerbasque, Basque Foundation for Science, E-48011 Bilbao, Spain
}

(Received 25 October 2019; accepted 7 February 2020; published 4 March 2020)

\begin{abstract}
Polarons are physical objects of material science that are hard to capture from first-principles calculations. $\mathrm{WO}_{3}$ is a paradigmatic system to study polarons and here we present calculations of a single self-trapped single polaron in $\mathrm{WO}_{3}$ from density functional theory calculations. Our calculations show that the single polaron is at a higher energy than the fully delocalized solution, in agreement with the experiments where a single polaron is an excited state of $\mathrm{WO}_{3}$. The symmetry-adapted mode decomposition of the polaron distortions shows that, among numerous modes, a polar zone center mode has the largest contribution and can be at the origin of the observed weak ferroelectricity of $\mathrm{WO}_{3}$.
\end{abstract}

DOI: 10.1103/PhysRevResearch.2.012052

Tungsten trioxide, $\mathrm{WO}_{3}$, is a wide band-gap semiconductor with a reference perovskitelike $\mathrm{ABO}_{3}$ structure, where the $A$ site is empty. Between 900 and $-180^{\circ} \mathrm{C}$, it undergoes a sequence of several structural phase transitions [1,2] involving the antipolar motion of $\mathrm{W}$ against $\mathrm{O}$ and different oxygen octahedra rotations $[3,4]$. Its ground state was reported to be ferroelectric but it was recently clarified that it should better a be nonpolar antiferroelectric insulator with the $P 2_{1} / c$ space group if perfectly stoichiometric [4].

$\mathrm{WO}_{3}$ is, however, disposed to be substoichiometric with oxygen vacancies $\left(\mathrm{WO}_{3-x}\right)$ or to host interstitial monovalent cations on its vacant $A$ site, driving numerous functional properties such as electrochromism [5-11]. The structural and electronic properties of $\mathrm{WO}_{3}$ are very sensitive to its dopant concentration $[12,13]$, which is directly connected with its propensity to form electronic polarons [13-18]. $\mathrm{WO}_{3}$ appears then to be a prototypical simple system to study electronic polarons and bipolarons, which are responsible for its rich optical properties [19].

Despite the numerous studies devoted to $\mathrm{WO}_{3-x}$, a full microscopic understanding of the underlying mechanisms responsible for its exceptional chromic responses is still lacking [20]. Previous studies based on density functional theory (DFT) or on simpler models have focused on the effect of oxygen vacancies [13,20-22] or of intercalated atoms such as $\mathrm{Li}$ or $\mathrm{Na}[22,23]$ but without a clear conclusion whether the extra electrons form localized small polarons around the

Published by the American Physical Society under the terms of the Creative Commons Attribution 4.0 International license. Further distribution of this work must maintain attribution to the author(s) and the published article's title, journal citation, and DOI. defects or if they can move as free carriers or form bipolaronic states [17]. While the former case of oxygen vacancies has been intensively studied from microscopic simulations, the case of free-carrier charge trapping has seldom been explored $[20,24]$ where it appeared that self-trapped electron polarons in $\mathrm{WO}_{3}$ could not be observed from DFT calculations.

In this Rapid Communication, we report a first-principles study of a self-trapped electron polaron in a bulk matrix of $\mathrm{WO}_{3}$ disconnected from its defect origin. Our results are in good agreement with the free-polaron electron density map of $\mathrm{W}^{5+}$ reported by Schirmer and Salje $[14,15,17]$ from their optical and electron spin resonance (ESR) measurements, where a single polaron has a two-dimensional (2D) disk shape extended on a few neighbors. The possibility to stabilize this $\mathrm{W}^{5+}$ state allows us to characterize its electronic and structural properties through real-space spin density, a density of states analysis, and a symmetry-adapted mode analysis of the atomic distortion of the crystal.

Our calculations have been carried out with the massively parallel version [25] of the CRYSTAL17 code [26] and the B1 $\mathrm{Wu}-$ Cohen (B1WC) hybrid exchange-correlation functional [27]. We have used the same Gaussian basis sets as in Ref. [4]. We have simulated the electron polaron by adding an additional electron into the system with charge neutrality being ensured by a compensating positively charged background.

These calculations have been performed on different supercell sizes where we found that a supercell made of $6 \times$ $6 \times 2$ reference cubic cells (one cubic unit cell containing four atoms, one $\mathrm{W}$ and three $\mathrm{O}$ ) is necessary to avoid too large interactions between periodic images and spurious longrange interaction effects [28]. We performed the structural relaxations with a supercell size and shape fixed to be the one of the $P 2_{1} / c$ ground-state phase of $\mathrm{WO}_{3}$ [4]. We stopped the relaxation when the root mean square on the force 


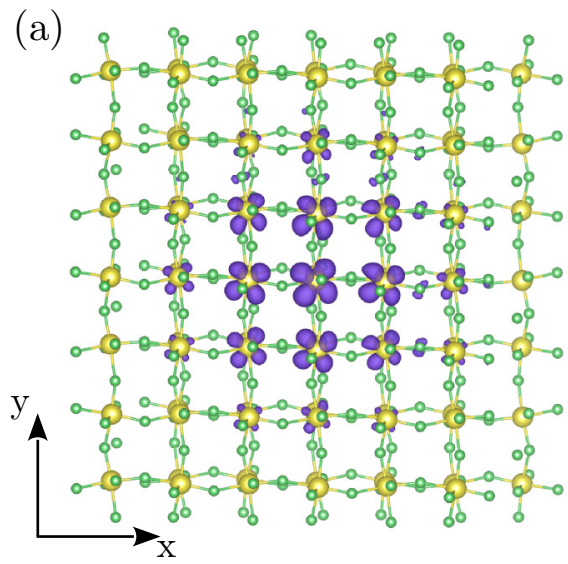

(b)

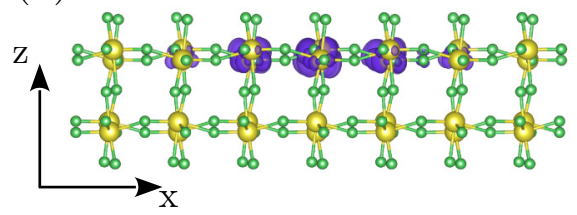

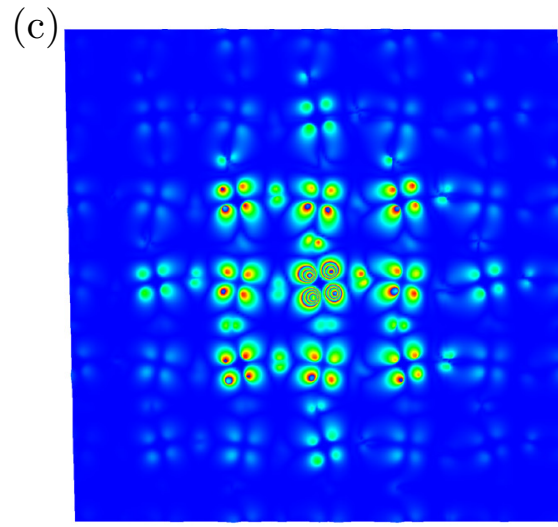

(d)

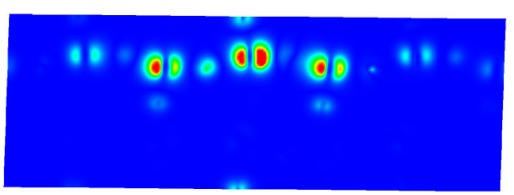

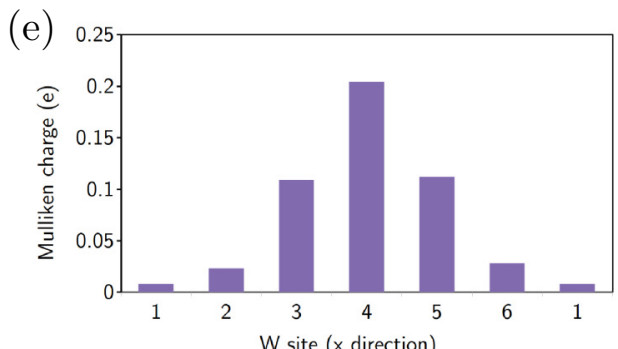

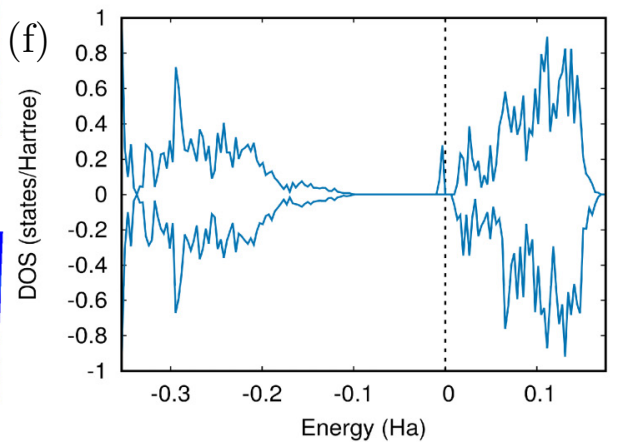

FIG. 1. 3D visualization of the calculated spin density (B1WC functional) of the polaron in (a) the $x y$ plane and (b) in the $x z$ plane of a $\mathrm{WO}_{3}$ supercell (W and $\mathrm{O}$ atoms are in yellow and green, respectively, and the charge density is in purple); (c) and (d) are associated 2D cut planes of the polaron density in the $x y$ and $x z$ planes passing through the central W atom where the charge localizes. (e) shows the Mulliken charge of $\mathrm{W}$ atoms along an $x$ line that goes through the central $\mathrm{W}$ in the $x y$ plane before and after atomic relaxation, respectively. (f) shows the density of states (DOS) around the Fermi level (dashed vertical line).

gradient and on the estimated displacements are smaller than $5 \times 10^{-5}$ hartree/bohrs and $1 \times 10^{-3}$ bohrs, respectively. The electronic self-consistent calculations were converged until the difference of the total energy is smaller than $10^{-8}$ hartree. The integration in the Brillouin zone has been performed on a $2 \times 2 \times 4$ grid of $k$ points in the $6 \times 6 \times 2$ supercell.

All the calculations were spin polarized and the overall magnetic moment has been constrained to be $1 \mu_{B}$ during the first relaxation steps to stabilize the ferromagnetic (FM) solution and the polaron localization. Once the full relaxation was done with this constraint, we released it without losing the polaron state, proving that this state is a stable local minimum. Another set of calculations have been done without constraining the magnetization of the cell, resulting in a nonmagnetic solution where the electron is fully delocalized. This shows that two key ingredients can be used to find the polaron state in $\mathrm{WO}_{3}$ : having realistic enough polaronic distortions as an initial guess and/or imposing the FM solution.

Let us present first the electronic structure characterizing the polaron, and discuss then its related atomic distortion. In Figs. 1(a)-1(d) we present the calculated real-space spinpolarization (or magnetization) density of the relaxed $6 \times 6 \times$ 2 supercell containing 288 atoms resulting from the addition of an electron into the $P 2_{1} / c$ phase of $\mathrm{WO}_{3}$. In Fig. 1(a) we show a top view of the $x y$ plane where we clearly see the localization of the extra electron density on a central $\mathrm{W}$ atom. Within our supercell size, this spin density also spreads mainly over the first, second, and third W neighbors as highlighted in the 2D plane going through the central $\mathrm{W}$ atom [Fig. 1(c)]. The shape of the density distribution corresponds to that of the W- $d_{x y}$ orbitals with four lobes pointing in the directions between the oxygen bonds and in agreement with the conduction-band bottom of $\mathrm{WO}_{3}$. The fact that the $d_{x y}$ orbitals are extended in two directions ( $x y$ ) could also explain the 2D shape of the polaron that is formed by the electron sitting in this $d_{x y}$ orbital. In Figs. 1(b) and 1(d) we report a side view of the spin density in the $x z$ plane where we can observe that the polaron charge localizes in one atomic plane of $\mathrm{WO}_{3}$ and does not spread in other atomic planes along the $z$ direction. We have also done a $4 \times 4 \times 4$ supercell, two times longer along the $z$ direction, and we did not find significant changes of the spread of the electron in the $z$ direction with respect to our $6 \times 6 \times 2$ supercell. This means that the density of the extra electron is anisotropic and forms a disk shape in the $x y$ plane. This disk shape of the electron density distribution is in good agreement with the one proposed by Schirmer and Salje $[14,17]$ from their ESR measurements of single excited polarons in $\mathrm{WO}_{3}$.

To complement the above qualitative picture, we report in Fig. 1(e) the value of the Mulliken charge of a selected chain of $\mathrm{W}$ atoms being on a line along $x$ passing through the central W. This Mulliken population analysis shows that $21 \%$ of the polaron electron density is localized on the central $\mathrm{W}$ with a strong decay when going away from it, resulting in a nearly zero value beyond the fourth neighbor. The localization obtained by our DFT calculations is smaller than the one reported experimentally, where $50 \%$ of the electron was reported to be present on the central atom $[14,17]$. This could be related to the exchange-correlation functional used where the amount of exact exchange can strongly influence the localization of the charge. 

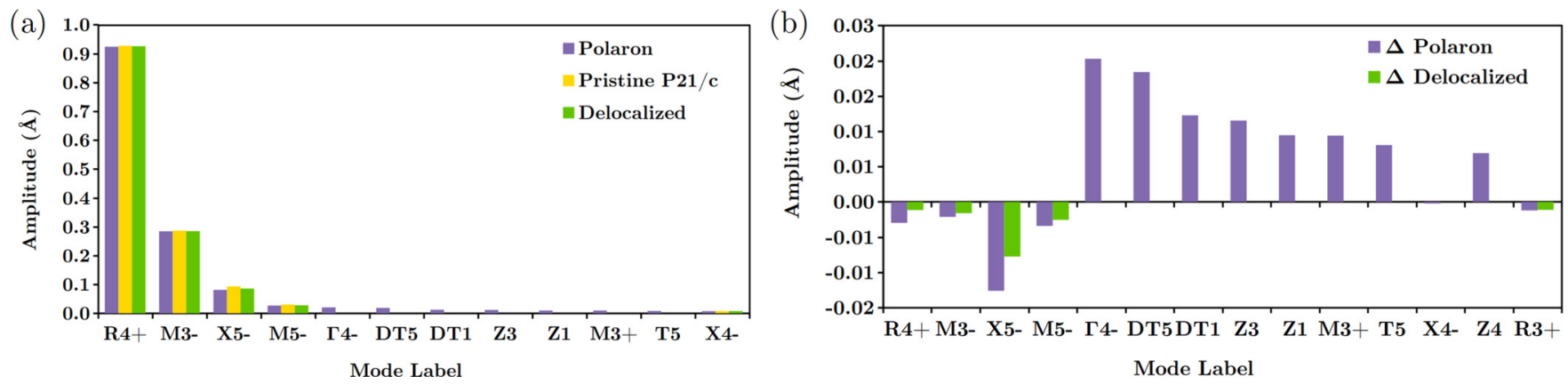

FIG. 2. (a) Symmetry-adapted mode analysis of the atomic distortions (in a $6 \times 6 \times 2$ supercell) between the reference cubic cell and the relaxed pristine $P 2_{1} / c$ ground state (yellow), the relaxed magnetic $P 2_{1} / c$ phase including a polaron (purple), and a relaxed nonmagnetic $P 2_{1} / c$ including a delocalized electron (green). In (b) we report the difference of mode amplitudes between the pristine $P 2_{1} / c$ phase and either the phase with a polaron (purple) or a delocalized electron (green). Only the modes with contributions larger than $0.009 \AA$ have been reported for clarity; the full decomposition can be found in the Supplemental Material [36] (Table S1 and Fig. S1). The $k$ vector of each mode is given in the Supplemental Material [36] (Table S1).

Hence, we obtain that the polaron is a medium polaron in the sense that its size is smaller than the large polarons observed in, e.g., $\mathrm{SrTiO}_{3}$ [29] or $\mathrm{CsPbBr}_{3}$ [30], but it does not correspond to what is defined as a small polaron, where the electron density is localized on the central atom and on the ligands around the defect [31]. This small extension of the electron polaron is compatible with the large anomalous Born effective charges and dielectric constant in $\mathrm{WO}_{3}$ [4], which, through a simple electrostatic interaction description, can explain screening of the charge of the polaron over short distances [21].

The self-trapping of a polaron is also known to be more important in strongly distorted crystals than in highly symmetric crystals. This effect is particularly striking in amorphous crystals where the trapping energies are larger than their crystalline counterpart [32] (up to $1.5 \mathrm{eV}$ for a hole polaron in, e.g., amorphous $\mathrm{Al}_{2} \mathrm{O}_{3}$ versus $0.13 \mathrm{eV}$ in its crystalline form [33]). It is also known that the crystal structure drives the shape of the polaron (2D polarons are favored in layered structures of $\mathrm{HfO}_{2}$ or $\mathrm{ZrO}_{2}$, for example [34]). The $P 2_{1} / c$ phase of $\mathrm{WO}_{3}$ has a low symmetry with dominant antipolar distortions [4]. This can account for the 2D shape of the polaron by lowering the $d_{x y}$ states with respect to the others. The calculations done on electron polarons in $\mathrm{SrTiO}_{3}$ [29] and halide perovskites [30] show them to be much bigger and delocalized (large polarons) than in $\mathrm{WO}_{3}$. Then, the stronger localization of the polaron in $\mathrm{WO}_{3}$ can be related to both large screening due to a large dielectric constant, and Born charges and to the stronger distortions present in the $P 2_{1} / c$ phase with respect to the other perovskites where polarons have been studied.

To further understand the electronic structure of our calculated polaron in $\mathrm{WO}_{3}$, we report in Fig. 1(f) the total density of states (DOS) of the supercell with the self-trapped polaron. We find that a localized state appears in the gap of $\mathrm{WO}_{3}$ in the up-spin channel [which is in agreement with the initial constraint of $1 \mu_{B}$ imposed at the beginning of the self-consistent-field (SCF) results of our calculations]. This state in the gap corresponds to the $d_{x y}$ state of $\mathrm{W}$ that slightly splits from the conduction bands (by about $0.4 \mathrm{eV}$ ), and it spreads over $0.1 \mathrm{eV}$ in the gap. Hence, it is not fully localized, i.e., in agreement with what we observe from the real-space density where the electron density of the polaron is spread over a few $\mathrm{W}$ atoms.

We conclude that our calculations allow us to simulate a self-trapped single polaron in the $P 2_{1} / c$ ground-state phase of $\mathrm{WO}_{3}$.

Now that we have characterized the electronic structure of the polaron in $\mathrm{WO}_{3}$, we proceed and analyze the impact of the self-trapping of the electron on the crystal structure. To clarify which type of displacements are involved in the crystal relaxation, we report in Fig. 2 the symmetry-adapted mode analysis (as obtained from the ISODISTORD software of the ISOTROPY software suite [35]) of the atomic distortions with respect to the reference cubic structures, (i) in the pristine $P 2_{1} / c$ phase (yellow bars, Fig. 2), (ii) in the $P 2_{1} / c$ phase with a polaron (purple bars in Fig. 2), and (iii) in a nonmagnetic $P 2_{1} / c$ phase with a fully delocalized extra electron (green bars in Fig. 2).

The first observation is that adding an extra electron distorts the $P 2_{1} / c$ bulk structure only slightly, either with the formation of the polaron or without it. As we can see in Fig. 2(b), where the differences in mode amplitudes between the pristine $P 2_{1} / c$ and the polaron and the delocalized solutions are plotted, the main effect of the polaron is to reduce all the bulk $P 2_{1} / c$ distortions, i.e., the octahedra rotations $\left(R_{4}^{+}\right)$and the antipolar motions $\left(M_{3}^{-}\right)$as well as the associated induced $X_{5}^{-}$and $M_{5}^{-}$modes from improper couplings with the $R_{4}^{+}$and $M_{3}^{-}$modes [4]. This is in line with what has been observed in lead halide perovskites where the hole polarons are observed to bring the structure closer to the cubic phase [30], thought this is quite a small effect in $\mathrm{WO}_{3}$.

We observe a similar reduction of the bulk $P 2_{1} / c$ distortions in both the polaron and the delocalized cases. This means that $n$ doping in $\mathrm{WO}_{3}$ has the general tendency to reduce the bulk distortions. This is in line with the experimental observation where, at high electron doping, $\mathrm{WO}_{3}$ changes completely its structure and adopts a tetragonal phase with distortions dominated by the Jahn-Teller effect of the $d^{1}$ orbital occupation [37] and becomes quite different from the $P 2_{1} / c$ phase. 
The self-trapping of the polaron induces numerous new additional modes, showing that the distortion is quite complex and can hardly be decomposed onto the basis of the phonon modes as observed also by Sio et al. [38,39] from perturbative approaches of electron polarons in other crystals. Over all of these extra modes, the first largest mode contribution is the polar mode $\Gamma_{4}^{-}$. This agrees with the proposition that a polaron in bulk $\mathrm{WO}_{3}$ can activate polar distortions due to its charge and may induce the weak ferroelectricity observed experimentally [4].

Our calculated self-trapped formation energy of the polaron (energy difference between the delocalized and polaron states) is about $+123 \mathrm{meV}$. Hence, we found that the formation energy is positive, meaning that the polaron state is higher in energy than the delocalized case and thus that the polaron forms a local metastable state of the crystal. This is somehow in agreement with the experimental reports where the singleelectron polaron state is obtained as an excited state of the system, the ground state being the bipolaronic state [17]. While we do not have access to the energy of the bipolaron due to supercell size limit, the fact that we found the single polaron being higher in energy than the fully delocalized state but nevertheless forming a local minimum in the energy landscape (if it would not form a local energy minimum, the calculations will always end up into the lowest-energy fully delocalized state once the FM constraint is removed) shows that a single polaron can be stabilized as an excited state of the crystal.

The fact that no self-trapping of the electron polaron in $\mathrm{WO}_{3}$ has been reported in previous DFT studies could come from several origins: the exchange-correlation functional used, the use of a more or less good initial guess, and also because the single polaron is not the ground state of bulk $\mathrm{WO}_{3}$.

The effect of the exchange correlation used has been reported in previous studies of $\mathrm{WO}_{3}$ where strong discrepancies between the Heyd-Scuseria-Ernzerhof hybrid (HSE06) (no possibility to stabilize a polaron away from defects) and the Becke three-parameter Lee-Yang-Parr functional (B3LYP) or HF15LYP (overlocalization of the polaron around defects) were observed [20,40]. Here, we used the B1WC hybrid functional, which includes the Wu-Cohen generalized gradient approximation (WC-GGA) correlation and mixes $16 \%$ of exact exchange with the WC-GGA exchange. It is surprising that our B1WC and previous HSE calculations give very similar results when comparing the bulk properties (band gap, crystal structure, etc.) while it seems not possible to obtain a single polaron with HSE as in B1WC. This might be related to the difference between hybrid functionals where only a fraction of exact exchange is added to the usual DFT functional (e.g., B1WC) and the range-separated hybrid functionals (e.g., HSE), which have an extra parameter to treat the long-range part. This could first mean that the DFT simulations of polarons in $\mathrm{WO}_{3}$ require specific attention with a fine tuning of the exchange-correlation functional parameters to reproduce correctly their formation (such as testing the piecewise linearity of the energy as fractional occupations [41-43]). This can, however, also be attributed to our extra FM constraint imposed in the first steps of the relaxation in the presence of the extra electron. This is supported by the fact that in the study of Wang et al. they tried to drive the trapping of the electron by setting some guessed initial distortions. Nevertheless, we observed in our case that doing so could not lead to the polaron formation if the starting distortions are not good enough. However, if one uses the FM solution constraint, then one can stabilize the polaron successfully, even if the initial guessed distortions are not so good. The FM constraint forces the calculation to go into the local energy well of the single localized electron. Then, once the polaron distortion is present, removing the FM constraint also leaves the polaron as a metastable state (local minimum), again proving that using an initial guess for the polaron distortion can be dramatic on the output simulation results for polarons.

In this Rapid Communication, we have showed the possibility to simulate from DFT calculations a self-trapped singleelectron polaron in the bulk $P 2_{1} / c$ phase of $\mathrm{WO}_{3}$, which forms a local minima of the energy surface and which is higher in energy than the fully delocalized state. This success is associated with the combination of an appropriate exchangecorrelation functional, a good initial guess of the polaronic distortions, and the imposition of a FM solution that can be released after the polaron is stabilized.

The self-trapped single-electron polaron that we obtained at higher energy is in good agreement with the excited single polaron reported experimentally where the polaron has a 2D shape with an extension over a few neighbors in one atomic plane. The quantitative results from the simulation (how spread/localized is the electron) are in reasonable agreement knowing that they are directly related to the exchangecorrelation functional used and, in the case of hybrid functionals, to the amount of exact exchange included. A detailed analysis should be done to determine the best exchange correlation to use in the case of $\mathrm{WO}_{3}$ to improve the quantitative agreement (e.g., piecewise linearity test [41-43]).

It would be interesting to use the recent reciprocal space perturbative formalism treatment of polarons of Sio et al. $[38,39]$ in the case of $\mathrm{WO}_{3}$ and to compare with our realspace analysis, if such an analysis could be applied to a local minimum that is not the ground state. Another point is that our calculations are done in the static limit, while the shape and lifetime of the polaron will be strongly influenced by the thermal and/or quantum fluctuations. Probing these effects from second-principles simulations $[44,45]$ would be interesting to further improve the qualitative picture of the simulated polarons.

We thank S. M. Casassa for her help with the CRYSTAL code and J. M. Perez Mato for discussions regarding the symmetry-adapted mode analysis. H.H., Ph.G., and E.B. acknowledge the FRS-FNRS, the Consortium des Equipements de Calcul Intensif (CECI), funded by the FRS-FNRS (Grants No. 2.5020.11 and No. 1175545), Tier-1 supercomputer of the Fédération Wallonie-Bruxelles funded by the Walloon Region (Grant No. 1117545), and the ARC project AIMED. E.K.H.S. thanks EPSRC (EP/P024904/1). E.A. acknowledges support by the Spanish Ministerio de Economía y Competitividad under Grant No. FIS2015-64886-C5-1-P. PAP acknowledges SFI-DfE (Grant No. 15/IA/3160).

E.B. and H.H. contributed equally to this work. 
[1] C. J. Howard, V. Luca, and K. S. Knight, J. Phys.: Condens. Matter 14, 377 (2002).

[2] K. R. Locherer, I. P. Swainson, and E. K. H. Salje, J. Phys.: Condens. Matter 11, 4143 (1999).

[3] M. Kawaminami and T. Hirose, J. Phys. Soc. Jpn. 46, 864 (1979).

[4] H. Hamdi, E. K. H. Salje, P. Ghosez, and E. Bousquet, Phys. Rev. B 94, 245124 (2016).

[5] S. K. Deb, Sol. Energy Mater. Sol. Cells 92, 245 (2008).

[6] G. A. Niklasson and C. G. Granqvist, J. Mater. Chem. 17, 127 (2007).

[7] G. A. Niklasson, L. Berggren, and A.-L. Larsson, Sol. Energy Mater. Sol. Cells 84, 315 (2004).

[8] C. G. Granqvist, Sol. Energy Mater. Sol. Cells 60, 201 (2000).

[9] C. Sella, M. Maaza, O. Nemraoui, J. Lafait, N. Renard, and Y. Sampeur, Surf. Coat. Technol. 98, 1477 (1998).

[10] Y. Zhao, Z. C. Feng, Y. Liang, and H. W. Sheng, Appl. Phys. Lett. 71, 2227 (1997).

[11] E. Salje, Opt. Commun. 24, 231 (1978).

[12] G. Mattoni, A. Filippetti, N. Manca, P. Zubko, and A. D. Caviglia, Phys. Rev. Materials 2, 053402 (2018).

[13] R. Chatten, A. V. Chadwick, A. Rougier, and P. J. D. Lindan, J. Phys. Chem. B 109, 3146 (2005).

[14] O. Schirmer and E. Salje, Solid State Commun. 33, 333 (1980).

[15] O. F. Schirmer and E. Salje, J. Phys. C 13, L1067 (1980).

[16] E. Salje and B. Güttler, Philos. Mag. B 50, 607 (1984).

[17] E. K. H. Salje, Eur. J. Solid State Inorg. Chem. 31, 805 (1994).

[18] A.-L. Larsson, B. E. Sernelius, and G. A. Niklasson, Solid State Ionics 165, 35 (2003).

[19] C. A. Triana, C. G. Granqvist, and G. A. Niklasson, J. Appl. Phys. 118, 024901 (2015).

[20] W. Wang, A. Janotti, and C. G. Van de Walle, J. Mater. Chem. C 4, 6641 (2016).

[21] N. Tsunoda, Y. Kumagai, M. Araki, and F. Oba, Phys. Rev. B 99, 060103(R) (2019).

[22] N. Bondarenko, O. Eriksson, and N. V. Skorodumova, Phys. Rev. B 92, 165119 (2015).

[23] S. Tosoni, C. D. Valentin, and G. Pacchioni, J. Phys. Chem. C 118, 3000 (2014).

[24] A. D. Walkingshaw, N. A. Spaldin, and E. Artacho, Phys. Rev. B 70, 165110 (2004).

[25] A. Erba, J. Baima, I. Bush, R. Orlando, and R. Dovesi, J. Chem. Theory Comput. 13, 5019 (2017).
[26] R. Dovesi, A. Erba, R. Orlando, C. M. Zicovich-Wilson, B. Civalleri, L. Maschio, M. Rérat, S. Casassa, J. Baima, S. Salustro, and B. Kirtman, Wiley Interdiscip. Rev.: Comput. Mol. Sci. 8, e1360 (2018).

[27] D. I. Bilc, R. Orlando, R. Shaltaf, G.-M. Rignanese, J. Íñiguez, and P. Ghosez, Phys. Rev. B 77, 165107 (2008).

[28] J. Bang, Y. Y. Sun, T. A. Abtew, A. Samanta, P. Zhang, and S. B. Zhang, Phys. Rev. B 88, 035134 (2013).

[29] M. Youssef, B. Yildiz, and K. J. Van Vliet, Phys. Rev. B 95, 161110(R) (2017).

[30] K. Miyata, D. Meggiolaro, M. T. Trinh, P. P. Joshi, E. Mosconi, S. C. Jones, F. De Angelis, and X.-Y. Zhu, Sci. Adv. 3, e1701217 (2017).

[31] A. L. Shluger and A. M. Stoneham, J. Phys.: Condens. Matter 5, 3049 (1993).

[32] J. Strand, M. Kaviani, D. Gao, A.-M. El-Sayed, V. V. Afanas'ev, and A. L. Shluger, J. Phys.: Condens. Matter 30, 233001 (2018).

[33] O. A. Dicks and A. L. Shluger, J. Phys.: Condens. Matter 29, 314005 (2017).

[34] K. P. McKenna, M. J. Wolf, A. L. Shluger, S. Lany, and A. Zunger, Phys. Rev. Lett. 108, 116403 (2012).

[35] ISOTROPY software suite, iso.byu.edu.

[36] See Supplemental Material at http://link.aps.org/supplemental/ 10.1103/PhysRevResearch.2.012052 for the full symmetry adapted mode decomposition.

[37] A. Aird, M. C. Domeneghetti, F. Mazzi, V. Tazzoli, and E. K. H. Salje, J. Phys.: Condens. Matter 10, L569 (1998).

[38] W. H. Sio, C. Verdi, S. Poncé, and F. Giustino, Phys. Rev. Lett. 122, 246403 (2019).

[39] W. H. Sio, C. Verdi, S. Poncé, and F. Giustino, Phys. Rev. B 99, 235139 (2019).

[40] F. Wang, C. Di Valentin, and G. Pacchioni, Phys. Rev. B 84, 073103 (2011).

[41] J. F. Janak, Phys. Rev. B 18, 7165 (1978).

[42] P. Erhart, A. Klein, D. Åberg, and B. Sadigh, Phys. Rev. B 90, 035204 (2014).

[43] G. Geneste, B. Amadon, M. Torrent, and G. Dezanneau, Phys. Rev. B 96, 134123 (2017).

[44] C. Escorihuela-Sayalero, J. C. Wojdeł, and J. Íñiguez, Phys. Rev. B 95, 094115 (2017).

[45] P. García-Fernández, J. C. Wojdeł, J. Íñiguez, and J. Junquera, Phys. Rev. B 93, 195137 (2016). 